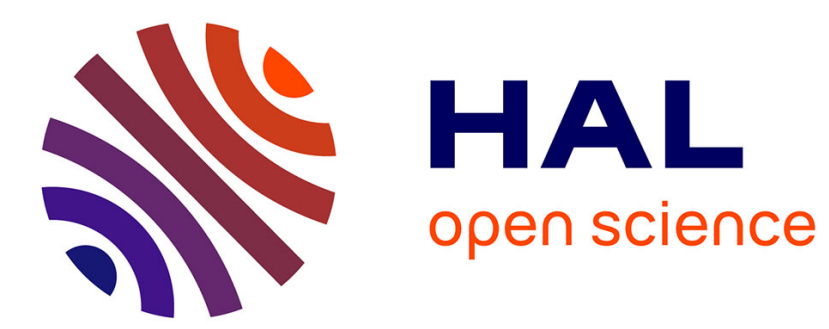

\title{
Evolution des propriétés tribocorrosives du 316L dans un milieu Nacl
}

\author{
G. Lederer, P. Ponthiaux, N. Celati, J. Vial, Christine Boher
}

\section{To cite this version:}

G. Lederer, P. Ponthiaux, N. Celati, J. Vial, Christine Boher. Evolution des propriétés tribocorrosives du 316L dans un milieu Nacl. Matériaux \& Techniques, 1997, n Hors Série, p. 25-27. 10.1051/mattech/199785070025s . hal-01851457

\section{HAL Id: hal-01851457 \\ https://hal.science/hal-01851457}

Submitted on 6 Nov 2019

HAL is a multi-disciplinary open access archive for the deposit and dissemination of scientific research documents, whether they are published or not. The documents may come from teaching and research institutions in France or abroad, or from public or private research centers.
L'archive ouverte pluridisciplinaire HAL, est destinée au dépôt et à la diffusion de documents scientifiques de niveau recherche, publiés ou non, émanant des établissements d'enseignement et de recherche français ou étrangers, des laboratoires publics ou privés. 


\title{
ÉVOLUTION DES PROPRIÉTÉS TRIBOCORROSIVES DU 316L DANS UN MILIEU NaCl
}

\author{
G. Lederer*, P. Ponthiaux**, N. Celati*, J. Vial*, C. Boher* \\ * DGA/DCE/CREA, Arcueil \\ ** Ecole Centrale Paris - Laboratoire CHF, Chatenay Malabry
}

$\mathrm{L}$

e frottement et l'usure des aciers inoxydables dans un environnement d'eau de mer est d'un intérêt primordial pour les applications navales. Si certaines études ont déjà été menées pour comprendre le rôle joué par les couches passives sur le frottement et l'usure des aciers inoxydables dans un milieu $\mathrm{H}_{2} \mathrm{SO}_{4}[1,2]$, peu sont consacrées au problème du frottement de ces matériaux dans l'eau de mer ou dans des électrolytes chlorurés [3]. Ces travaux se sont principalement intéressés à l'effet d'un environnement corrosif sur l'usure et l'influence des caractéristiques mécaniques a souvent été sous-estimée.

L'objectif de cette étude est de corréler les mesures électrochimiques et d'usure avec les paramètres du contact et les propriétés mécaniques de l'acier inoxydable $316 \mathrm{~L}$ dans une solution de $\mathrm{NaCl}$ (A3). L'évolution du potentiel libre sous frottement a été étudiée et une explication de son comportement global est proposée en intégrant des informations mécaniques et électrochimiques.

\section{Procédure expérimentale}

Le moyen expérimental est un tribomètre de type pion sur disque rotatif. Le pion est en rotation selon un rayon de $8 \mathrm{~mm}$. La configuration du contact choisie est celle d'un pion à bout hémisphérique d'un rayon de $30 \mathrm{~mm}$ sur un disque plan, ceci permettant d'évaluer les pressions de contact. La charge appliquée pour toutes les expériences est de $40 \mathrm{~N}$ et, afin de limiter les effets thermiques, la vitesse de rotation est de $40 \mathrm{tr} / \mathrm{min}$. Toutes les expériences sont menées à température ambiante $\left(21^{\circ} \mathrm{C}\right)$ dans une solution aqueuse renouvelée $(\mathrm{NaCl}$ à $3 \%$ tamponné à $\mathrm{pH} 8)$. L'électrode de référence utilisée pour les mesures électrochimiques est une électrode $\mathrm{Ag} / \mathrm{AgCl}$. Tous les potentiels sont donnés par rapport à celle-ci. Pour les expériences sous contrôle potentiostatique, une contre-électrode circulaire en platine est utilisée. Cette géométrie a été choisie de manière à fournir une polarisation acceptable de la surface exposée de l'échantillon. Les courbes de polarisation sont déterminées avec une vitesse de balayage de $30 \mathrm{mV} / \mathrm{min}$.

Le pion est en $\mathrm{Al}_{2} \mathrm{O}_{3}$ fritté (module d'élasticité de $400 \mathrm{GPa}$ ). Le disque de $316 \mathrm{~L}$ présente un module élastique de $207 \mathrm{GPa}$ et une limite élastique en traction de $225 \mathrm{MPa}$; la pression hertzienne maximale $p_{0}$ est alors de $578 \mathrm{MPa}$. Dans ces conditions, le contact est en régime de lubrification limite.

Les tests d'usure sous contrôle potentiostatique incluent deux étapes: une polarisation de 15 min avant frottement, puis frottement continu durant 5 heures. L'usure finale $W$ est déterminée à l'aide de mesures profilométriques de la trace d'usure.

Conférence prononcée au colloque Eurocorr'96 Le coefficient de frottement est obtenu en divisant la force de frottement par la force normale appliquée, ces deux forces étant mesurées en continu. Chaque échantillon uti(session Tribocorrosion), Nice, 24-26 septembre 1996. 
chaque potentiel imposé $(-1500,-800, \ldots+300 \mathrm{mV})$ pour assurer une stabilité correcte des forces de frottement. Les valeurs du coefficient de frottement rapportées sont la moyenne des valeurs obtenues dans la partie stationnaire de la courbe pour chaque palier de potentiel.

\section{Résultats}

\section{Comportement électrochimique sans frottement}

Les courbes de polarisation obtenues (fig. 1) sont en accord avec les mesures faites dans un milieu d'eau de mer naturelle [4]. Cependant, elles diffèrent de celles obtenues dans un électrolyte de type $\mathrm{H}_{2} \mathrm{SO}_{4}$ : absence du pic d'activation, transition passivité/piqûration au lieu de passivité/transpassivité et présence d'une densité de courant cathodique résiduelle inférieure à $10 \mu \mathrm{A} / \mathrm{cm}^{2}$ dans la région de passivité (attribuée à la présence d'oxygène dissout). Le potentiel en circuit ouvert $E_{\text {cor }}$ évolue autour de $-180 \mathrm{mV}$ et le potentiel de protection autour de $0 \mathrm{mV}$.

\section{Evolution du potentiel libre sous frottement}

Dans nos conditions expérimentales, le frottement déplace la valeur du potentiel libre vers des valeurs plus cathodiques, aux alentours de $-400 \mathrm{mV}$. Ce type de comportement est similaire à celui observé dans des solutions d'acide sulfurique

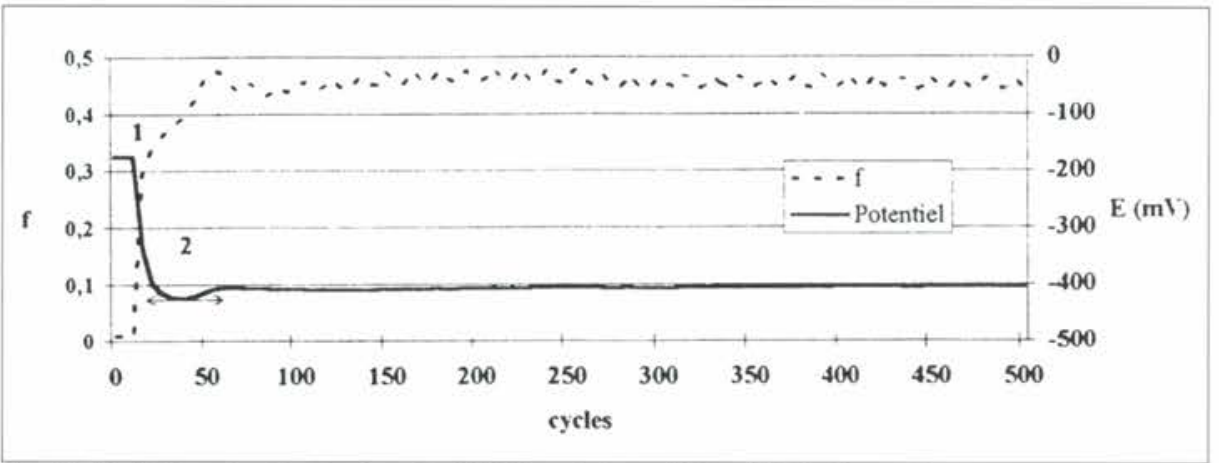

Fig. 2 - Evolution simultanée du potentiel libre et du coefficient de frottement.

[1] et montre la sensibilité du potentiel à des perturbations par frottement de la surface. Deux domaines peuvent être distingués (fig. 2): une période transitoire d'environ 50 cycles suivie d'une période stationnaire.

\section{Frottement et usure sous contrôle potentiostatique}

Chaque point expérimental des figures 3 et 4 est le résultat de trois expériences et les barres d'erreur correspondent à \pm l'écarttype associé à la répétabilité des expériences. Elles ont été déterminées par une méthode statistique d'analyse de la variance [5].

La figure 3 montre que le coefficient de frottement est très sensible au potentiel appliqué, ceci étant particulièrement vrai autour de la valeur du potentiel libre sous frottement $(-400 \mathrm{mV})$ : une petite variation autour du potentiel imposé sous frottement conduit à des variations significatives des forces de frottement. Cependant, les points extrêmes de la figure 3 correspondent à des conditions électrochimiques particulières : réduction d'hydrogène importante à $-1500 \mathrm{mV}$ et corrosion par piqûration à $+300 \mathrm{mV}$.

Pour toutes ces expériences, aucune particule d'usure macroscopique n'est observée sur les échantillons ou dans la solution filtrée. Inversement, la présence de produits de corrosion est manifeste et son importance dépend du potentiel imposé.

La figure 4 présente en échelles lin-log le volume d'usure dans les régions de passivation et de piqûration. Celui-ci est nettement influencé par le potentiel mais l'usure totale reste faible. Pour un potentiel de $+300 \mathrm{mV}$, les piqûres de corrosion observées sont distribuées de façon homogène sur toute la surface y compris dans la trace d'usure. Le pion en alumine n'a pas été affecté par le frottement et aucun transfert n'a été observé, quelque soit la plage de potentiels.

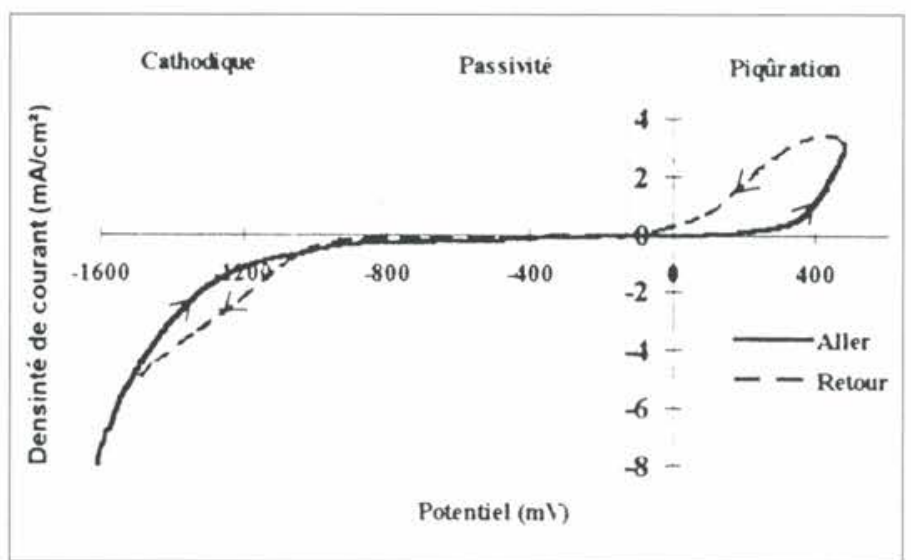

Fig. 1 - Courbe de polarisation en l'absence de frottement du $316 \mathrm{~L}$ dans $\mathrm{NaCl}$ à $3 \% \mathrm{pH} 8$.

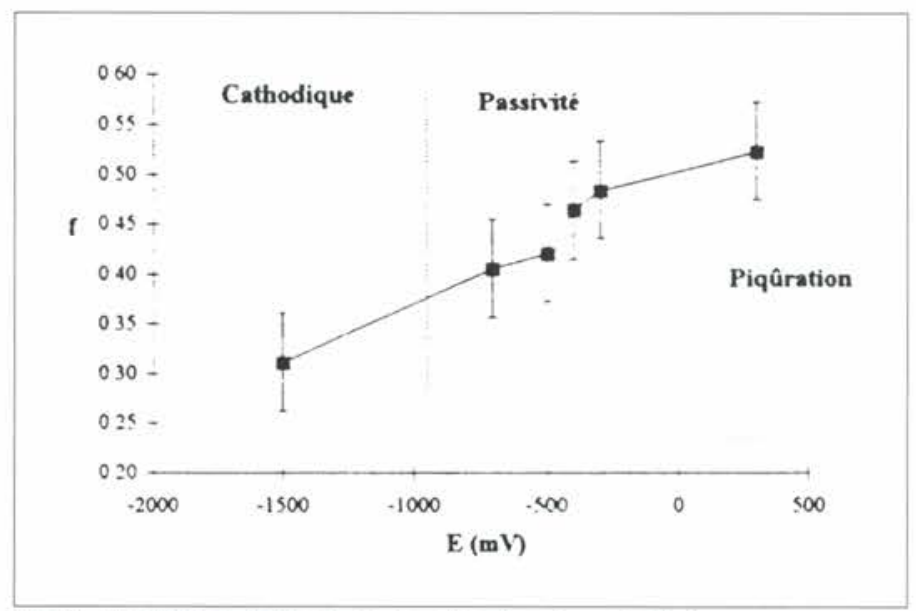

Fig. 3 - Coefficient de frottement en fonction du potentiel.

Fig. 4 - Volume d'usure total $W$ en fonction du potentiel.

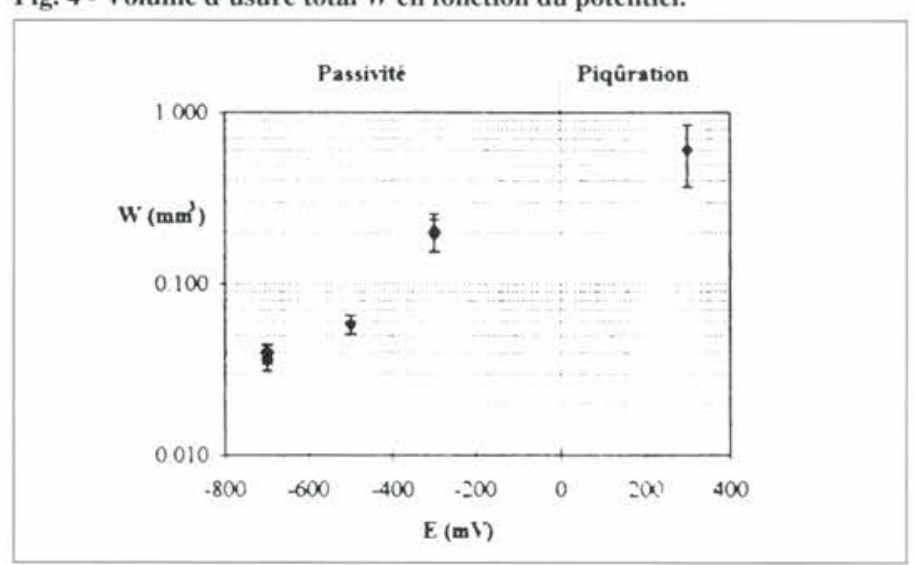




\section{Discussion}

Afin de proposer une explication des résultats électrochimiques et d'usure, une analyse succincte de l'état mécanique du contact peut s'avérer utile.

Le $316 \mathrm{~L}$ présente un comportement élasto-plastique cinématique non-linéaire et isotrope [6]. La limite élastique initiale en cisaillement. $k$, est de $144 \mathrm{MPa}$. Une analyse hertzienne du contact [7] prévoit une pression maximale au centre de 4,2 $k$. Cette valeur, associée à des coefficients de frottement supérieurs à 0,3 , signifie suivant ce modèle que des déformations plastiques devraient avoir lieu à chaque cycle en surface. Ceci implique donc que même sans usure, la surface affectée par le frottement va augmenter du fait de la plastification.

Par conséquent, une interprétation de la période transitoire (fig. 2) peut être proposée.

Le potentiel libre est le résultat d'un équilibre entre les courants résultants des réactions anodiques et cathodiques distribuées sur la surface. Soit $\theta$ (resp. $(1-\theta)$ ), le taux de recouvrement des réactions anodiques (resp. cathodiques) avec une densité de courant $j_{a}(E)$ (resp. $j_{c}(E)$ ). Le comportement typique de ces densités de courant est schématisé figure 5. Au potentiel libre avant frottement, nous avons:

$$
\theta j_{a}+(1-\theta) j_{c}=0
$$

Cette relation reste vraie (le courant global mesuré reste nul) y compris lors de l'application du frottement.

On obtient donc en différentiant par rapport au temps :

$$
\frac{d \theta}{d t}=\frac{j_{c} \frac{d J_{a}}{d E}-j_{a} \frac{d J_{c}}{d E}}{\left(J_{a}-J_{c}\right)^{2}} \cdot \frac{d E}{d t}=A(E) \cdot \frac{d E}{d t}
$$

et on montre aisément que $\mathrm{A}(\mathrm{E})<0 \quad \forall E$ [8]. La variation du potentiel libre est donc opposée à celle de $\theta$. Le frottement va

dégrader les propriétés passives des couches de surface soit par amincissement soit par ablation [2], augmentant ainsi la surface anodique. Quand le frottement débute, $d \theta / d t$ est donc positif et $d E / d t$ est négatif, ce qui est en accord avec le

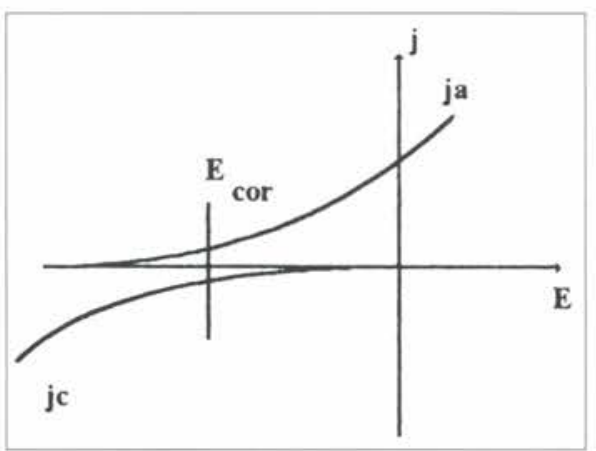

Fig. 5 - Equilibre à $E_{\text {cor }}$. saut de potentiel observé (fig.2, repère 1).

L'évolution lente de $E$ dans la zone transitoire peut être attribuée aux changements de la géométrie du contact dus aux incréments de déformations plastiques à chaque passage du pion. Après le changement de signe de $d E / d t$ (fig. 2, repère 2), l'amplitude de ces incréments va décrôttre jusqu'à atteindre un état stationnaire pouvant être purement élastique. En effet, la présence de déformations plastiques au cours du régime stationnaire conduirait à une usure par fatigue plastique [7] caractérisée par la présence de débris macroscopiques. Aucune particule de ce type n'étant observée après plus de $10^{4}$ cycles, nous supposons donc que le régime stabilisé est élastique, ce point devant être confirmé par une approche numérique appropriée [9].

La sensibilité des forces de frottement au potentiel appliqué est, quant à elle, plus délicate à interpréter. Une analyse par SIMS des couches superficielles n'a pas permis de faire apparaître de différences significatives sur la nature de ces couches en fonction du potentiel.

La dépendance en potentiel du volume d'usure (fig. 4) fait l'objet de travaux en cours incluant la sensibilité des phénomènes de dissolution/passivation au potentiel appliqué.

\section{Conclusion}

L'influence des paramètres électrochimiques sur les propriétés de quelques systèmes tribologiques a été étudiée (sensibilité des forces de frottement et de l'usure au potentiel dans la zone passive).
L'évolution globale des essais fait apparaître, après une période transitoire dépendant des propriétés mécaniques, une période stationnaire qui dépend des propriétés électrochimiques de la surface (usure corrosive).

\section{RÉFÉRENCES}

[1] XX. Jiang, S.Z. Li, D.D. TAO, J.X. Yang, Corr. Sci., 49, 836-841 (1993).

[2] S. Mischler, E.A. Rosset, D. Landolt, Thin Film Tribology, 245-253, D. Dowson et al. ed., Elsevier Amsterdam (1993).

[3] Y. YAhagi and Y. MizUtani, Wear, 110, 401-408 (1986).

[4] C. Compère, P. Jaffre, D. Festy, Corrosion 95, paper 289, NaCE, Houston Tx (1995).
[5] Commissariat à I'Energie Atomique, Statistiques appliquées à l'exploitation des mesures, vol. I et II, Masson, Paris (1978).

[6] P CORDIER, Thèse ENPC, Paris (1986).

[7] K.L. JoHnSON, Wear, 190, 162-170 (1995).

[8] P. Ponthiaux, G. Lederer, N. Celati, C. Boher, C. Batalllon, Stage COMETT, St Martial de Nabirat (1993)

[9] K. Dang Van, H.M. Maitournam, J. Mech. Phys. Solids, 48, 11 , (1993). 